Acta Crystallographica Section D

Biological Crystallography

ISSN 0907-4449

\title{
Outrunning free radicals in room-temperature macromolecular crystallography
}

A significant increase in the lifetime of room-temperature macromolecular crystals is reported through the use of a highbrilliance X-ray beam, reduced exposure times and a fastreadout detector. This is attributed to the ability to collect diffraction data before hydroxyl radicals can propagate through the crystal, fatally disrupting the lattice. Hydroxyl radicals are shown to be trapped in amorphous solutions at $100 \mathrm{~K}$. The trend in crystal lifetime was observed in crystals of a soluble protein (immunoglobulin $\gamma$ Fc receptor IIIa), a virus (bovine enterovirus serotype 2) and a membrane protein (human $\mathrm{A}_{2 \mathrm{~A}}$ adenosine $\mathrm{G}$-protein coupled receptor). The observation of a similar effect in all three systems provides clear evidence for a common optimal strategy for roomtemperature data collection and will inform the design of future synchrotron beamlines and detectors for macromolecular crystallography.

Structural Biology, The Henry Wellcome Building for Genomic Medicine, Roosevelt Drive, Oxford OX3 7BN, England, ${ }^{\mathbf{d}} \mathrm{NIHR}-$ Leeds Musculoskeletal Biomedical Research Unit and Leeds Institute of Molecular Medicine, University of Leeds, Leeds LS9 7FT, England,

$\mathbf{e}_{\text {Heptares Therapeutics Ltd, BioPark, Welwyn }}$ Garden City AL7 3AX, England, and ' MRC Laboratory of Molecular Biology, Hills Road, Cambridge CB2 OQH, England

Correspondence e-mail:

robin.owen@diamond.ac.uk
(C) 2012 International Union of Crystallography Printed in Singapore - all rights reserved
Received 26 January 2012

Accepted 22 March 2012

\section{Introduction}

The cryocooling of crystals in macromolecular crystallography (MX) greatly increases their lifetime in the X-ray beam, and for this reason the vast majority of synchrotron-based MX is performed using crystals held at $100 \mathrm{~K}$ in an open-flow nitrogen cryostat (Garman \& Owen, 2007). Despite the advantages provided by cryocooling, there is still considerable interest in carrying out room-temperature (RT) crystallography at synchrotron sources, and this has been reflected by the development of dedicated sample environments at several facilities (Jacquamet et al., 2004; Bingel-Erlenmeyer et al., 2011; Axford et al., 2012). The reasons for this interest are twofold. Firstly, screening of crystals in situ removes the invasive and potentially destructive step of crystal mounting and eliminates confounding factors such as cryoprotection and crystal handling when establishing optimal crystallization conditions. Secondly, some macromolecules, in particular viruses, prove to be difficult or indeed impossible to successfully cryocool, precluding data collection at $100 \mathrm{~K}$. Additional motivation for collecting data at room temperature comes from recent work suggesting that data collection at $100 \mathrm{~K}$ can hide conformational diversity (Fraser et al., 2011). In both cases radiation damage becomes a limiting factor during data collection and it is often impossible to collect a complete data set from a single crystal, or indeed a small number of crystals.

Several systematic radiation-damage studies at $100 \mathrm{~K}$ have shown no, or little, dose-rate effect (Garman, 2010). Accrued damage is only a function of the dose absorbed by the sample, and not of the manner in which it is deposited. In contrast, RT studies using both rotating-anode and synchrotron sources 
have shown a significant variation in crystal lifetime as a function of the rate at which dose is deposited in the crystal (Blake \& Phillips, 1962; Southworth-Davies et al., 2008; Rajendran et al., 2011; Warkentin et al., 2011). A dose-rate effect has also recently been observed at $260 \mathrm{~K}$ (Warkentin et al., 2012). These observations are consistent with datacollection times being comparable with the timescales of $\mathrm{X}$-ray-induced chemical reactions and raise the exciting possibility of significantly increasing the amount of data that can be collected from a crystal by changing the way in which data are collected. The work reported here aims to address two questions that have yet to be answered fully. Firstly, what dose-rate effects exist when RT macromolecular crystals are exposed to the very high dose rates $\left(\sim 1 \mathrm{MGy} \mathrm{s}^{-1}\right)$ accessible at undulator beamlines and, secondly, on what timescales do dose-rate effects become significant?

Recent advances in detector technology have resulted in a paradigm change in the way data can be collected at MX beamlines. Until recently, the de facto standard was a CCD capable of reading out a single frame every $1-2 \mathrm{~s}$, although this maximum frame rate is not realised at many beamlines owing to the need to ready the goniometer between images. We refer to this method of data collection as 'stop-start'. The advent in MX of large-area pixel-array detectors (PADs; Eikenberry et al. 2003) and, more recently, fast CCDs capable of reading out up to 30 frames per second (fps) dramatically changes the timescales on which it is possible to collect diffraction data, allowing so-called shutterless, or continuous, data collection. Continuous data collection truly opens a new temporal dimension in crystallography, potentially allowing the collection of diffraction data before damage can accumulate in, or propagate through, the sample. Damage has been observed to progress during the time following exposure to X-rays when the X-ray shutter is closed (Blundell \& Johnson, 1976; Warkentin et al., 2011): the absence of a pause during data collection eliminates this dark progression of damage, potentially allowing the collection of more data from a given sample. Continuous data collection enables for the first time ready investigation of the diffractive properties of protein crystals on subsecond timescales. The combination at I24, Diamond Light Source of a high-brilliance beamline providing $\sim 10^{12}$ photons s $^{-1}$ into $\sim 10 \times 10 \mu \mathrm{m}$ and a fast-readout detector provides the opportunity to investigate RT radiation damage in weakly diffracting macromolecular crystals on short timescales.

In the work described here, the X-ray-induced decay of protein and virus crystals has been monitored as a function of dose rate and detector-readout mode in order to establish whether increased lifetimes result (i) when diffraction data are collected continuously without repeated pauses in X-ray irradiation while the detector reads out and (ii) when data are collected with increased incident fluxes for a shorter period of time, breaking the linear relationship between absorbed dose and accrued damage observed at cryogenic temperatures. The identity of the free radicals responsible for the difference in the radiation susceptibility of macromolecular crystals has been probed using UV-Vis absorption spectroscopy.

\section{Methods}

\subsection{Crystallization}

Immunoglobulin $\gamma \mathrm{Fc}$ receptor IIIa (Fc $\gamma$ RIIIa) crystals were grown as described previously (Axford et al., 2012) and were loop-mounted within a capillary during data collection. Crystals of $20-30 \mu \mathrm{m}$ in size grew in space group $P 6_{1} 22$, with unit-cell parameters $a=b \simeq 60.6, c \simeq 214 \AA$, in $0.5 \mathrm{M} \mathrm{NaCl}$, $0.1 M$ sodium citrate tribasic dyhydrate, $2 \%(v / v)$ ethylene imine polymer $\mathrm{pH}$ 5.5. Bovine enterovirus serotype 2 (BEV 2) crystals of $\sim 30 \mu \mathrm{m}$ in size were grown in space group $F 23$, with unit-cell parameters $a=b=c \simeq 436.6 \AA$, in $1.5 M$ ammonium sulfate, $0.1 M$ bis-tris propane $\mathrm{pH} 7.0$ using the sitting-drop method (Walter et al., 2005). Diffraction data were collected directly from crystals within the crystallization tray using the in situ setup available at I24. Crystals of the $\mathrm{A}_{2 \mathrm{~A}}$ adenosine Gprotein coupled receptor $\left(\mathrm{A}_{2 \mathrm{~A}} \mathrm{AR}\right)$ were grown in space group $C 2$, with unit-cell parameters $a=76.5, b=98.9, c=79.5 \AA$, in $0.05 M$ Tris-HCl pH 7.6, 9.6\% PEG 200, 22.9\% PEG 300 as described by Lebon et al. (2011) and were loop-mounted within a capillary for data collection.

\subsection{Diffraction data collection}

Diffraction data were collected on the microfocus beamline I24 at Diamond Light Source. The beam was defocused to $20 \times 20 \mu \mathrm{m}$ at the sample position to match the typical crystal size, with the focal plane of the beam shifted towards the detector by $40 \mathrm{~mm}$. Data were collected at X-ray energies of $12.68 \mathrm{keV}$ (Fc $\gamma$ RIIIa crystals) and $12.8 \mathrm{keV}$ (BEV 2 and $\mathrm{A}_{2 \mathrm{~A}} \mathrm{AR}$ crystals). Diffraction data were recorded using a Pilatus 6M PAD (Dectris) modified to run at frame rates of up to $25 \mathrm{~Hz}$. Diffraction data were collected in two experimental modes: 'stop-start' and 'continuous'. During stop-start data collection the experimental shutter was closed for a 4,8 or $12 \mathrm{~s}$ period between consecutive images, emulating a traditional MX experiment in which the detector takes a few seconds to read out and the hardware must be positioned in preparation for the next image. Owing to software and hardware limitations at the time of the experiments, a pause of less than $4 \mathrm{~s}$ was not possible. During continuous data collection the X-ray shutter remained open for the duration of the experiment, which is the default mode of operation for PADs on the Diamond MX beamlines.

$\mathrm{X}$-ray diffraction data were analysed using LABELIT (Sauter \& Poon, 2010). Using DISTL, the integrated signal strength given in pixel ADC units above the local background of all Bragg candidates was calculated on a per-image basis. This was defined as the diffracting power of the crystal, allowing rapid determination of the diffracting power as a function of image number. The dose in MGy absorbed by each crystal during a data set was determined using RADDOSE (Paithankar \& Garman, 2010). Dose calculations showed that the crystals of Fc $\gamma$ RIIIa, BEV 2 and $\mathrm{A}_{2 \mathrm{~A}} \mathrm{AR}$ were subject to maximum dose rates of $0.69,0.89$ and $1.00 \mathrm{MGy} \mathrm{s}^{-1}$, respectively. In order to establish whether a dose-rate effect existed, data were collected with a range of exposure times from 0.04 to $4.09 \mathrm{~s}$, with the beam being attenuated such that 
the dose absorbed per image was constant, i.e. no attenuation was used for exposure times of $0.04 \mathrm{~s}$, while the beam was attenuated by $98.5 \%$ for $4.09 \mathrm{~s}$ exposures. All intensity decays were fitted with an exponential function of the form $I=a+$ $b \exp \left(\right.$ dose $\left./ d_{e}\right)$. The quantity $d_{e}$ represents the dose required for the diffracting power of the crystal to fall by 1/e and was taken as the crystal lifetime. To compare directly with other studies, in particular those carried out at $100 \mathrm{~K}$, where a linear intensity decay is observed and the dose required for the diffracting power to fall by half is quoted, the lifetimes here should be multiplied by $2 / e$.

The Pilatus $6 \mathrm{M}$ is a paralyzable detector: for a dead-time period after each photon is recorded a detector pixel is 'blind' to incident X-rays. A correction is applied to the counts recorded by the detector to account for this. The combination of high fluxes and short exposure times used in these experiments mean that a count-rate correction may be significant for some reflections and, in the limit, would result in systematic underestimation of intensities. Appendix $A$ and Supplementary Fig. S1 ${ }^{1}$ show that even for the most intense reflections observed from Fc $\gamma$ RIIIa, $A_{2 A} A R$ and BEV 2 the dose-rate correction is small and does not invalidate the model used.

\subsection{UV-Vis data collection}

In situ UV-Vis absorption spectra were collected on beamline I02 at Diamond Light Source. Spectra were collected using mirror lenses (Bruker) mounted in an off-axis geometry and a deuterium halogen light source (Ocean Optics). Spectra were recorded over the wavelength range of 200-750 nm using a Shamrock 303 imaging spectrograph (Andor). Data were collected using an X-ray energy of $12.658 \mathrm{keV}$, an X-ray beamsize of $70 \times 110 \mu \mathrm{m}$ and a UV-Vis focal spot $100 \mu \mathrm{m}$ in diameter. Spectra were collected using an exposure time of $40 \mathrm{~ms}$ with eight accumulations per spectrum written to disk.

\section{Results}

The high doses required to record useful diffraction from the samples used in this study resulted in extremely rapid crystal decay. Crystal lifetimes can therefore only be determined over a small number of images $(<25)$, resulting in some apparent variation in lifetime between crystals subjected to the same incident flux. To address this, and in order that overall trends in lifetime as a function of dose rate can be determined without crystal-to-crystal variation being a dominant factor, a large number of crystals have been used in this study $(>110)$. All crystals exhibited an exponential decay in intensity and an exponential function with an $R$ value greater than 0.9 could be fitted to the observed decay. It is important to note that the ability to record only a small number of images does not preclude structure solution: the structures of Fc $\gamma$ RIIIa and BEV 2 have both been solved using RT data recorded in situ at I24 (Axford et al., 2012).

\footnotetext{
${ }^{1}$ Supplementary material has been deposited in the IUCr electronic archive (Reference: TZ5006). Services for accessing this material are described at the back of the journal.
}

In order to investigate slow (timescales of $\geq 1 \mathrm{~s}$ ) damage accumulation, diffraction data were collected from Fc $\gamma$ RIIIa crystals in both stop-start and continuous data-collection modes. Continuous data were collected with exposure times per frame of $0.09 \mathrm{~s}(11 \mathrm{~Hz}), 1 \mathrm{~s}$ and $4.09 \mathrm{~s}(0.24 \mathrm{~Hz})$, while stop-start data were collected with an exposure time of $0.09 \mathrm{~s}$ and a pause of 4,8 or $12 \mathrm{~s}$, emulating and exaggerating typical CCD shuttered data-collection modes used over the last 15 years at synchrotron beamlines. An additional stop-start data

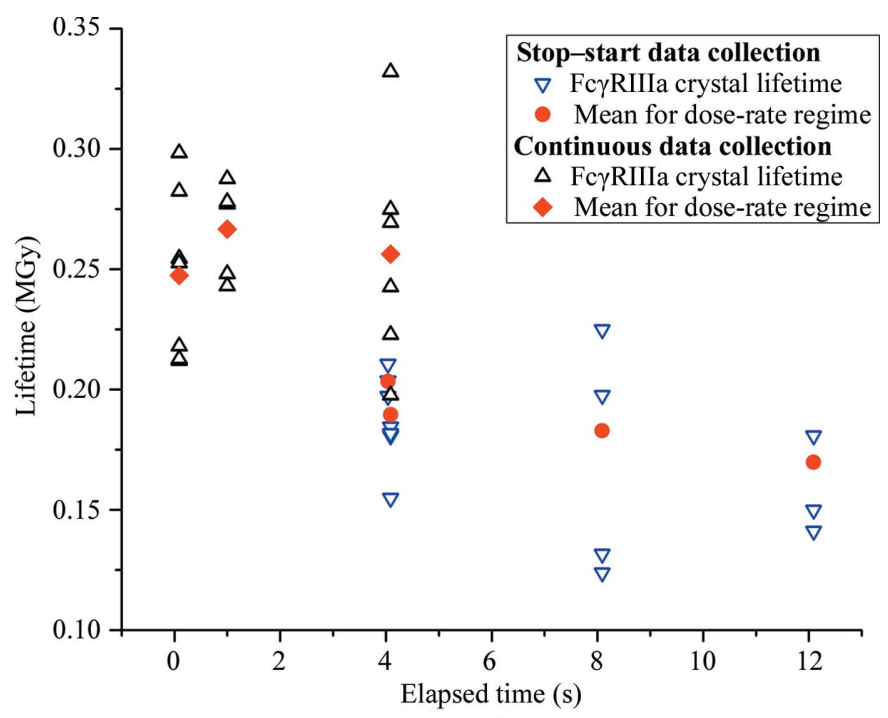

(a)

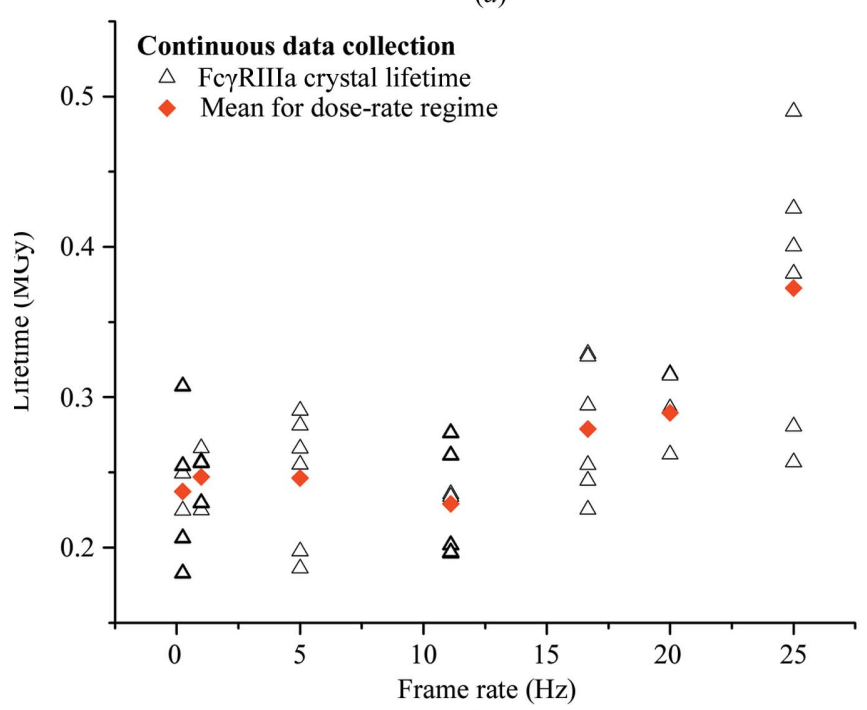

(b)

Figure 1

Comparison of Fc $\gamma$ RIIIa crystal lifetimes in both stop-start and continuous data-collection modes. Data sets when the fast shutter was closed between images are termed 'stop-start' and are shown as blue open triangles, with the mean for each regime shown as a red circle. Data sets when the detector was read out continuously are shown as filled black triangles, with the mean shown as a red diamond (top). For all data, elapsed time is equal to the exposure time plus the shutter closed time between images ( $0 \mathrm{~s}$ in the case of continuous data collection). The bottom panel shows the lifetime of Fc $\gamma$ RIIIa crystals as a function of frame rate. All data were collected in continuous data-collection mode, i.e. with the X-ray shutter left open for the duration of the experiment. Data for individual crystals are shown as black triangles and the means for each dose-rate regime are shown as red diamonds. 
series was also collected with an exposure time of $0.04 \mathrm{~s}$ and a pause of $4 \mathrm{~s}$. The incident beam was attenuated so that the absorbed dose per image was the same for each dose-rate regime. All data are summarized in Fig. 1(a). A systematically shorter crystal lifetime was observed for data collected using a stop-start approach (mean lifetime 0.186 MGy) in comparison to continuous data (mean lifetime $0.257 \mathrm{MGy}$ ). Within each data-collection mode there is very little variation in lifetime.

Shorter timescales were probed in Fc $\gamma$ RIIIa crystals by collecting data continuously with exposure times of 4.09$0.04 \mathrm{~s}$, corresponding to frame rates of $0.24-25 \mathrm{~Hz}$ (Fig. $1 b$ ). Below $11 \mathrm{~Hz}$ (exposure times $\geq 0.09 \mathrm{~s}$ ) the crystal lifetime is

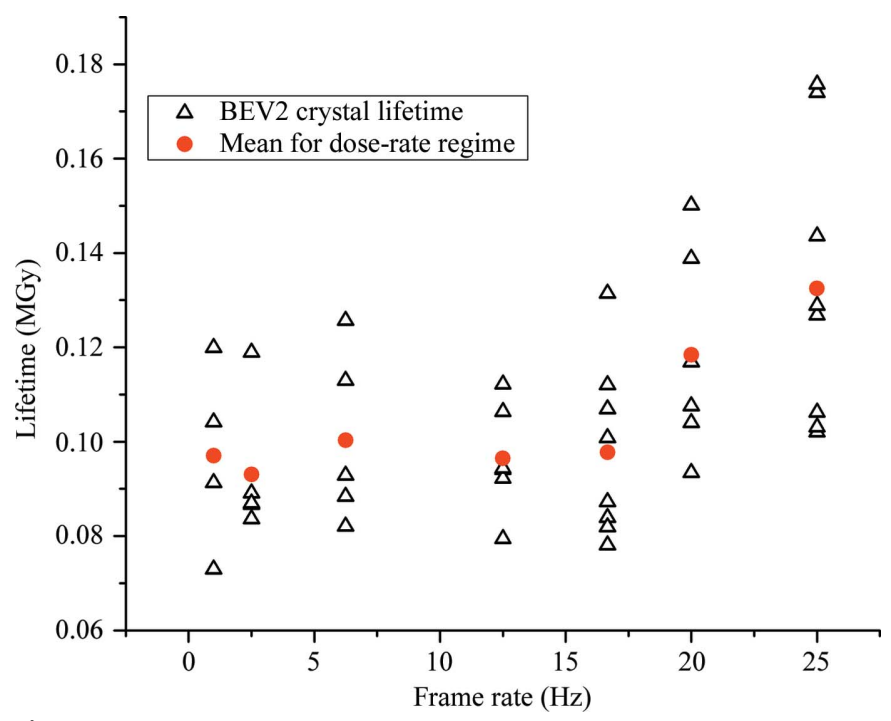

Figure 2

Plot showing variation in the lifetime of BEV crystals as a function of frame rate. At frame rates above $15 \mathrm{~Hz}$ an increase in the mean lifetime of the crystals is apparent. The dose per frame is kept constant for all crystals and frame rates.

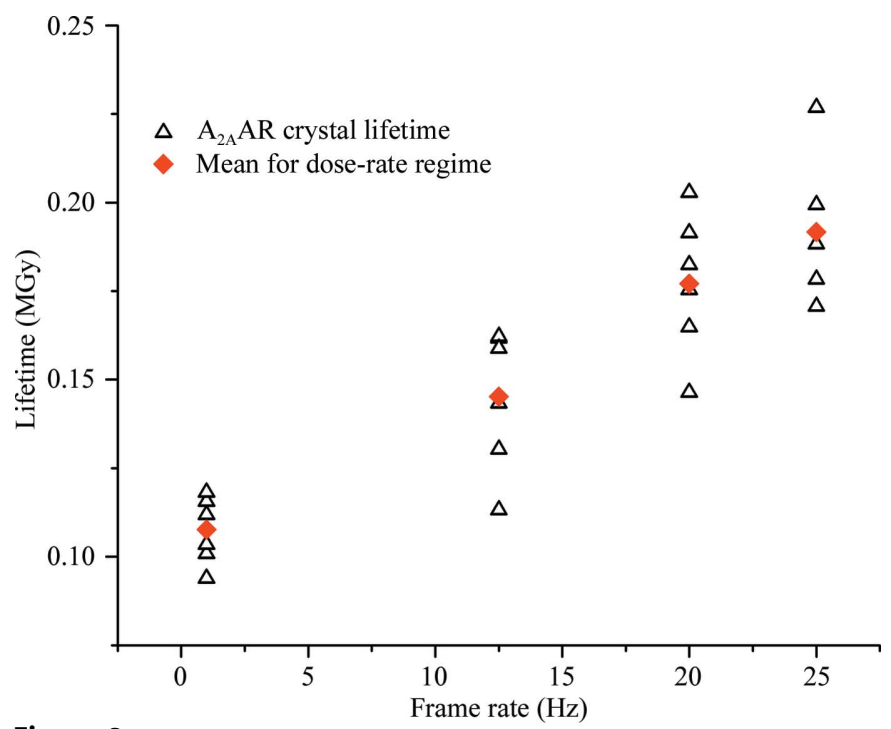

Figure 3

Plot showing variation in the lifetime of $\mathrm{A}_{2 \mathrm{~A}} \mathrm{AR}$ crystals as a function of frame rate. A linear relationship between frame rate and lifetime can be observed. The dose per frame is kept constant for all crystals and frame rates. almost constant as a function of dose rate, but at higher frame rates (shorter exposure times) a systematic increase in lifetime becomes apparent. Below $11 \mathrm{~Hz}$ the mean lifetime is $0.257 \mathrm{MGy}$; when data are collected at $25 \mathrm{~Hz}$ (exposure time $40 \mathrm{~ms}$ ) this increases to $0.373 \mathrm{MGy}$. This represents a significant increase on the mean lifetime of 0.186 MGy observed in the stop-start experiments.

The continuous exposure dose-rate experiments were repeated on BEV 2 crystals and a similar trend in crystal lifetime was observed (Fig. 2). In the case of BEV 2 the crystal lifetime was observed to be approximately constant up to frame rates of $16 \mathrm{~Hz}$ (exposure times $\geq 0.06 \mathrm{~s}$ ), with a mean crystal lifetime of $0.097 \mathrm{MGy}$. At higher frame rates the mean lifetime increased, reaching $0.132 \mathrm{MGy}$ at $25 \mathrm{~Hz}$. The experimental approach for data collection from BEV 2 crystals

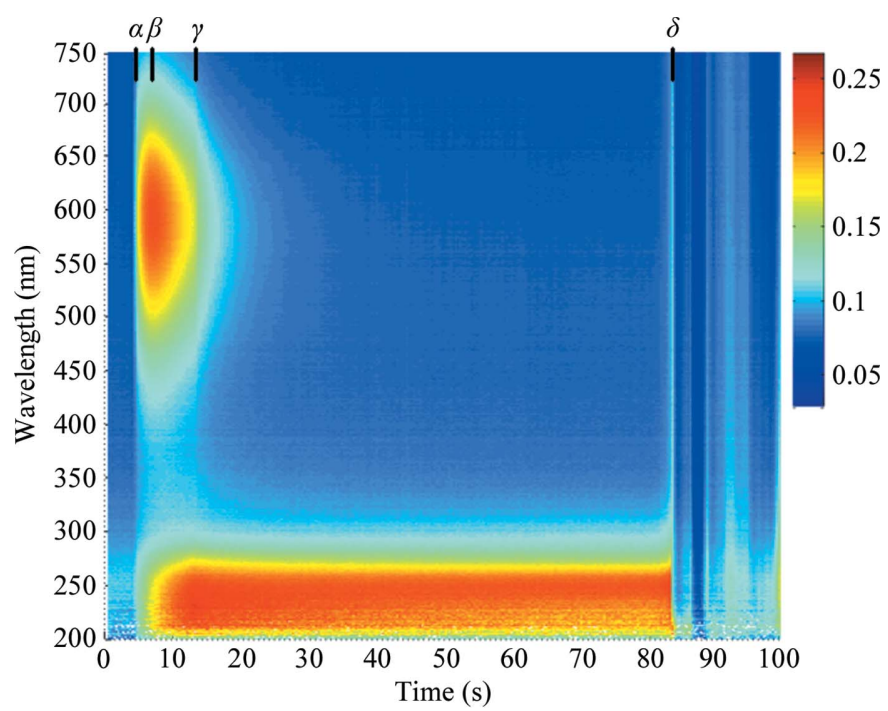

(a)

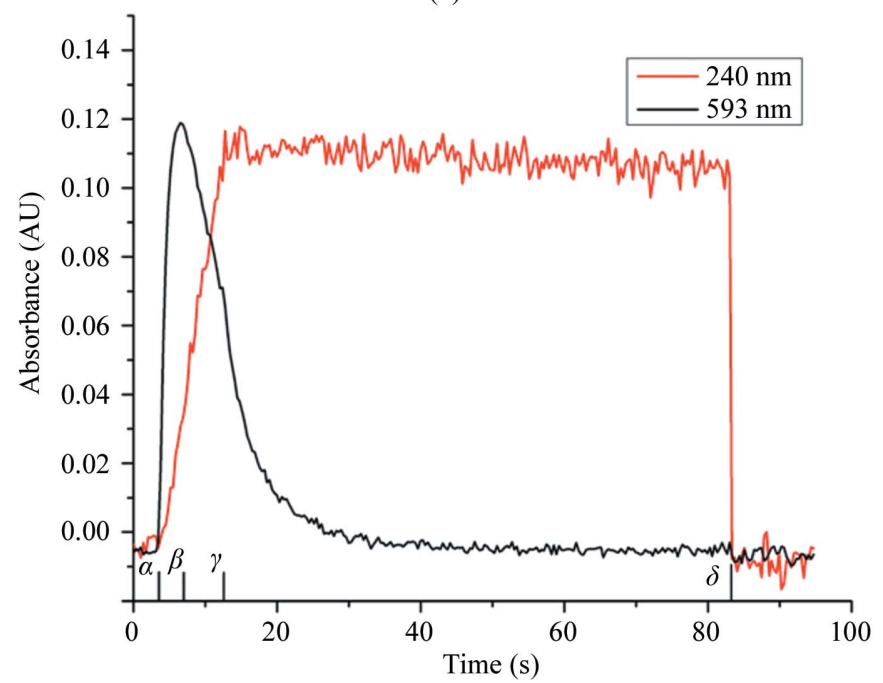

(b)

Figure 4

Change in the UV-Vis absorption spectra of Fc $\gamma$ RIIIa crystallization solution at $100 \mathrm{~K}$ upon exposure to X-rays. (a) shows a contour plot showing changes at all wavelengths between 200 and $750 \mathrm{~nm}$, while $(b)$ shows the change in absorbance at 240 and $593 \mathrm{~nm}$. The X-ray shutter is opened at $\alpha(t=3.40 \mathrm{~s})$, the $593 \mathrm{~nm}$ peak reaches a maximum at $\beta(6.50 \mathrm{~s})$, the X-ray shutter is closed at $\gamma(12.40 \mathrm{~s})$ and the $100 \mathrm{~K}$ nitrogen stream is blocked at $\delta(83 \mathrm{~s})$. 
differed in that data were collected from crystals in the crystallization drop rather than loop-mounted within a capillary. The methodology of the experiment was otherwise identical.

Continuous exposure dose-rate experiments were also carried out on crystals of $\mathrm{A}_{2 \mathrm{~A}} \mathrm{AR}$. However, owing to the limited availability of crystals fewer dose-rate regimes could be probed. In contrast to Fc $\gamma$ RIIIa and BEV 2, the lifetime of adenosine $A_{2 A} A R$ crystals varied almost linearly as a function of dose rate, increasing from $0.108 \mathrm{MGy}$ at $1 \mathrm{~Hz}$ to $0.192 \mathrm{MGy}$ at $25 \mathrm{~Hz}$ (Fig. 3). While a clear increase in lifetime as a function of dose rate is apparent, the lack of dose-rate regimes probed make it impossible to establish whether a region with no dose-rate effect exists at low frame rates.

UV-Vis absorption data were collected from thin films of Fc $\gamma$ RIIIa well solution held at $100 \mathrm{~K}$. Before exposure to $\mathrm{X}$-rays the spectra showed no features and were flat over the wavelength range $200-750 \mathrm{~nm}$ (Fig. 4a). Upon exposure to $\mathrm{X}$-rays a peak centred at $593 \mathrm{~nm}$ rapidly evolved and followed a first-order exponential decay (Fig. 4b). A second peak at $240 \mathrm{~nm}$ grew at a slower rate and remained present until the $100 \mathrm{~K}$ nitrogen stream was blocked and the sample temperature was allowed to rise. The transient peak at $593 \mathrm{~nm}$ is indicative of the generation of aqueous, or solvated, electrons (McGeehan et al., 2009), while we postulate that the peak at $240 \mathrm{~nm}$ is representative of the hydroxyl radical, as observed elsewhere (Hug, 1981; Janik et al., 2007). Upon slower warming of $\mathrm{Fc} \gamma \mathrm{RIII}$ a well solution the absorbance at $240 \mathrm{~nm}$ was observed to remain approximately constant until $\sim 160 \mathrm{~K}$ (Fig. 5). Irradiation of thin films of sodium citrate alone, a principal component of the Fc $\gamma$ RIIIa well solution, resulted in absorption peaks at 263 and $332 \mathrm{~nm}$ with no peak at $240 \mathrm{~nm}$.

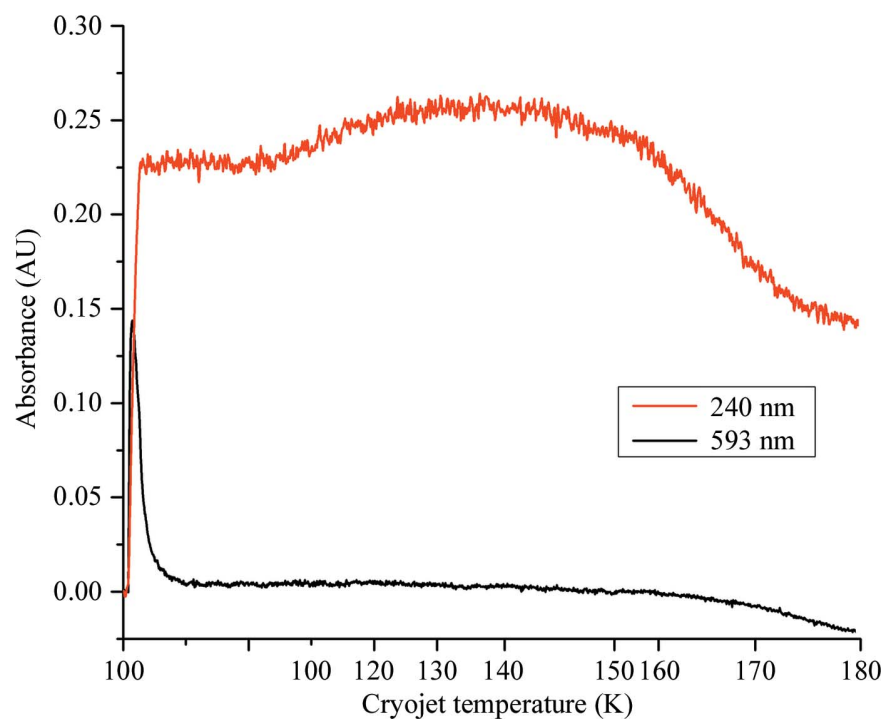

Figure 5

Change in UV-Vis absorbance of Fc $\gamma$ RIIIa crystallization solution at 240 and $593 \mathrm{~nm}$ irradiated at $100 \mathrm{~K}$ and then warmed to $180 \mathrm{~K}$. The $x$ axis reflects the readback temperature from the cryojet controller; the temperature at the sample will differ from this. Note that this axis is nonlinear and approximate. At approximately $150 \mathrm{~K}$ absorbance at low wavelengths decreases significantly, indicating increased mobility of radicals. The absorbance at $240 \mathrm{~K}$ does not return to zero owing to discolouration and icing of the sample upon slow warming.
UV-Vis absorption data were also collected from thin films of pure water, but the presence of microcrystalline ice made the collection of low-wavelength $(<250 \mathrm{~nm})$ data impossible. Nonetheless, a clear absorbance shoulder was observed to form at the low-wavelength limit upon exposure to X-rays, indicating the formation of hydroxyl radicals (Appendix $B$ ). UV-Vis absorption data were also collected from thin films of Fc $\gamma$ RIIIa well solution at RT: the spectra showed no change upon exposure to X-rays. A low-wavelength absorbance peak was also observed to form upon irradiation of solutions containing glycerol, but in this case an increase in absorbance at $240 \mathrm{~nm}$ is likely to indicate the breakdown of glycerol to malonic dialdehyde (Ivanova et al., 2009).

In order to compare the dose-rate effects in Fc $\gamma$ RIIIa, BEV and adenosine $\mathrm{A}_{2 \mathrm{~A}} \mathrm{AR}$ crystals, mean lifetimes are overlaid in Fig. 6. To facilitate comparison, the Fc $\gamma$ RIIIa lifetimes have been divided by a factor of two, so that in all cases the crystal lifetime at $1 \mathrm{~Hz}$ is of the order of $0.1 \mathrm{MGy}$. The plot clearly shows a dose-rate effect in all systems studied, with increased crystal lifetimes observed when collecting data continuously at high frame rates $(\geq 16 \mathrm{~Hz})$.

\section{Discussion}

This study provides clear evidence for a dose-rate effect in RT macromolecular crystallography when using the full flux of an undulator beamline at a third-generation synchrotron in conjunction with a fast-readout detector. This study extends previous work on RT data collection and radiation damage (Cherezov et al., 2002; Southworth-Davies \& Garman, 2007; Barker et al., 2009; Rajendran et al., 2011; Warkentin et al., 2012). The Fc $\gamma$ RIIIa, BEV 2 and $A_{2 A} A R$ crystals used in this study were subjected to maximum dose rates of 689,886 and $995 \mathrm{kGy} \mathrm{s}^{-1}$, which are comparable to the maximum

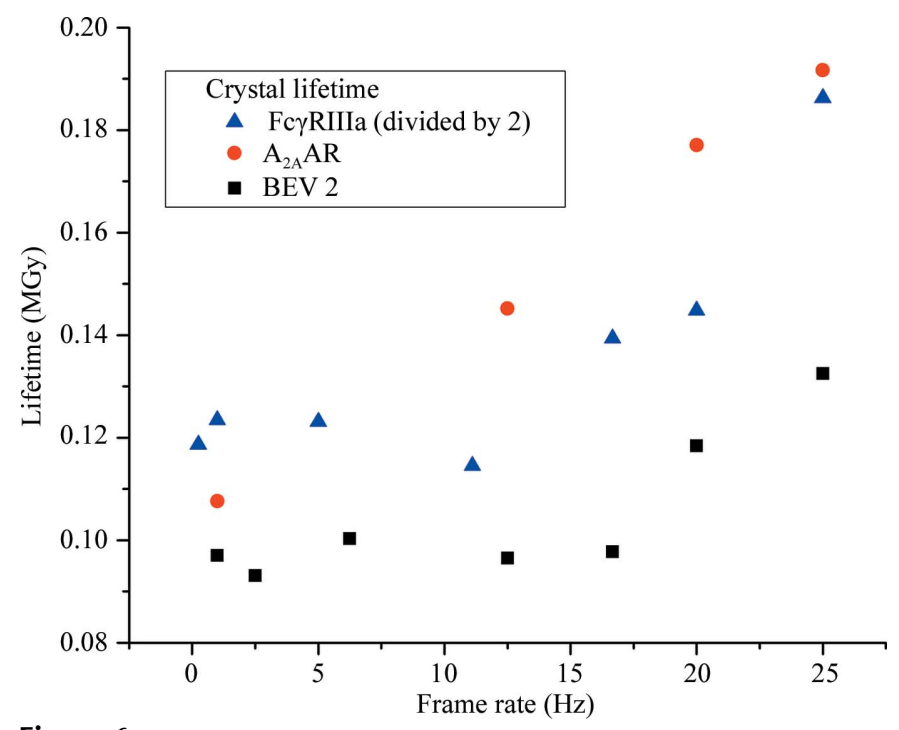

Figure 6

Lifetime of Fc $\gamma$ RIIIa, BEV and $\mathrm{A}_{2 \mathrm{~A}} \mathrm{AR}$ crystals as a function of dose. Note that the Fc $\gamma$ RIIIa data have been scaled by a factor of two to facilitate comparison with the BEV 2 and $\mathrm{A}_{2 \mathrm{~A}}$ data. All crystal types show an increase in lifetime at higher frame rates. 
previously reported (Warkentin et al., 2012). In addition, the fast-readout detector enabled 25 fps to be collected. This is almost two orders of magnitude beyond previous studies with CCDs and image plates. At exposure times of less than $60 \mathrm{~ms}$ (>16 fps) lifetimes increase as a function of dose rate. We also found that the crystal lifetime reduced by a factor of two when there was a pause between frames within a data set when, for example, the X-ray detector is read out, in contrast to previous findings (Warkentin et al., 2012). Despite differences in experiment design between this and other studies, it is interesting to note the similarity in the crystal lifetimes observed. RT lifetimes are of a similar magnitude, i.e. $\sim 0.2 \mathrm{MGy}$, and are approximately two orders of magnitude less than lifetimes at $100 \mathrm{~K}$, suggesting that the processes that dominate roomtemperature X-ray-induced damage are essentially complete on timescales shorter than even those studied here.

What are these processes? On very short timescales it has been demonstrated (using an X-ray free-electron laser with an $\mathrm{X}$-ray pulse length of a few tens of femtoseconds) that very intense X-ray beams allow a massive X-ray dose to be deposited, leading to measurable diffraction from crystals at RT before the complete ionization of the structure destroys the lattice in less than 100 fs (Neutze et al., 2000; Chapman et al., 2011). In contrast, the processes in our experiments occur over timescales some ten orders of magnitude longer in crystals subjected to a much more modest dose rate.

At the X-ray energies used in macromolecular crystallography, photoelectric absorption accounts for most of the energy $(\sim 84 \%)$ deposited in the crystal. Following such an interaction, atoms predominantly relax through ejection of an Auger electron, resulting in positive holes, and free electrons with high kinetic energy which are unlikely to return to the parent atom (Paithankar et al., 2009). Owing to the high solvent content of macromolecular crystals $^{2}$ much of the photoelectric absorption results in direct or indirect radiolysis of water, resulting in the formation of aqueous (solvated) electrons $\left(\mathrm{e}_{\mathrm{aq}}^{-}\right)$and hydroxyl radicals $(\cdot \mathrm{OH})$ (Klassen, 1987; Symons, 1999),

$$
\mathrm{H}_{2} \mathrm{O} \stackrel{h v}{\longrightarrow} \mathrm{H}_{2} \mathrm{O}^{+}+\mathrm{e}_{\text {dry }}^{-} \stackrel{\mathrm{H}_{2} \mathrm{O}}{\longrightarrow} \mathrm{H}_{3} \mathrm{O}^{+}+\cdot \mathrm{OH}+\mathrm{e}_{\mathrm{aq}}^{-}
$$

(Klassen, 1987). Approximate yields can be estimated through comparison with experiments carried out under anaerobic conditions (Sclavi et al., 1997). For every $100 \mathrm{eV}$ of energy absorbed under anaerobic conditions, the following numbers of radicals are obtained: $4.14 \mathrm{H}_{2} \mathrm{O}, 2.7 \mathrm{H}^{+}, 2.7 \mathrm{e}_{\text {aq }}^{-}$and $2.87 \cdot \mathrm{OH}$ (Buxton, 1987). The mobility of radicals, and hence their ability to propagate through a unit cell and cause damage, changes significantly as a function of temperature. Hydrogen is mobile at temperatures above $\sim 30 \mathrm{~K}$ (Mao et al., 2002). Interestingly, cooling protein crystals to below this temperature can have a deleterious effect. Trapped hydrogen results in a loss of short-range order, outweighing any gain resulting from a reduction in free-radical mobility (Meents et al., 2010). Electrons and holes are mobile in proteins at temperatures

\footnotetext{
${ }^{2}$ For the crystals used in this study, the solvent contents are 63,83 and $69 \%$ for Fc $\gamma$ RIIIA, BEV and $A_{2 A} A R$, respectively.
}

down to $77 \mathrm{~K}$ (Jones et al., 1987; Symons, 1995), suggesting that the large difference in the rates of damage at $100 \mathrm{~K}$ and RT does not arise from these species. In contrast, the mobility of $\cdot \mathrm{OH}$ is believed to change significantly between $100 \mathrm{~K}$ and RT. The ESR spectra of ice and poly(ethylene oxide) show .OH to be trapped at $77 \mathrm{~K}$ (Brivati et al., 1969; Zakurdaeva et al., 2005) and upon warming to $115 \mathrm{~K}$ (Zakurdaeva et al., 2005 ) to $130 \mathrm{~K}$ (Symons, 1999) $\cdot \mathrm{OH}$ becomes mobile and any spectroscopic signature disappears.

At $100 \mathrm{~K}$ aqueous electrons can move through the unit cell and rapidly react, with the result that their spectroscopic signature rapidly reaches a maximum (at $\sim 45 \mathrm{kGy}$; Owen et al., 2011) and then decays (Fig. 4). Hydroxyl radicals remain trapped and are unable to react, so the characteristic $\cdot \mathrm{OH}$ band does not decay (Fig. 4). Upon warming of solutions to room temperature the $\cdot \mathrm{OH}$ band disappears in less than $0.3 \mathrm{~s}$. At RT it was not possible to observe the formation of either aqueous electron or hydroxyl peaks, although the samples rapidly became discoloured. Upon controlled warming of Fc $\gamma$ RIIIa solutions the $\cdot \mathrm{OH}$ band remained constant up to temperatures of $\sim 160 \mathrm{~K}$. Above this temperature absorbance at $240 \mathrm{~nm}$ decreased, suggesting increased radical mobility, but did not return to zero. This may arise from discolouration of the sample, icing or the formation of further radical products which absorb at this wavelength. The change in mobility of . OH between $100 \mathrm{~K}$ and room temperature indicated by these results together with previous studies suggest that $\cdot \mathrm{OH}$ radicals are the root cause of greatly increased rates of damage in RT crystallography.

For these experiments, samples were either loop-mounted within a capillary ( $\mathrm{Fc} \gamma \mathrm{RIII}$ and $\left.\mathrm{A}_{2 \mathrm{~A}} \mathrm{AR}\right)$ or held in situ within a crystallization tray (BEV 2) at $295 \mathrm{~K}$ for data collection. In the absence of active cooling by a gas stream it might be expected that X-ray-induced sample heating is significant and has an effect on crystal lifetime. Measurement of the temperature rise induced in a glass bead exposed to $\sim 3 \times 10^{12}$ photons s $^{-1}$ (energy $6.5 \mathrm{keV}$, beamsize $103 \times$ $84 \mu \mathrm{m})$ in the absence of a gas stream revealed a temperature rise of $\sim 25 \mathrm{~K}$ over $5 \mathrm{~s}$ (Snell et al., 2007). The observed temperature rise over $1 \mathrm{~s}$ was somewhat lower: $\sim 10 \mathrm{~K}$. Taking the differing X-ray energy used, the significantly larger X-ray attenuation length and the smaller size of the protein crystals into account, it can be assumed that these observations provide an upper bound for the temperature rise in a protein crystal (Snell et al., 2007). While large temperature rises may result from prolonged X-ray exposure, the above combined with the brief duration of the experiments described here means that temperature effects were not considered to be a critical factor determining crystal lifetime.

How do $\cdot \mathrm{OH}$ radicals cause damage? They are extremely reactive, with their rate of reaction being diffusion-limited (Dorfman \& Adams, 1973; Swartz \& Swartz, 1983; Xu \& Chance, 2007). This reactivity is exploited in the field of footprinting, in which $\cdot \mathrm{OH}$ radicals are used to cleave nucleic acid or protein chains (Sclavi et al., 1997; Xu \& Chance, 2007). In proteins, a number of reaction pathways are possible: mainchain cleavage may occur either directly through attack at the 
$\alpha$-carbon or proline oxidation or by radical transfer via side chains. Hydroxyl interaction via side chains is 10-10 000 times faster than with the backbone (Takamoto \& Chance, 2006). The solvent composition has a significant effect on the efficiency of footprinting: buffers such as Tris, citrate, HEPES, MOPS, CAPS and CAPSO increase the lifetime owing to their unfavourable (in the case of footprinting) $\cdot \mathrm{OH}$-quenching properties, whereas sodium cacodylate or phosphate buffers have been reported to have minimal quenching properties $(\mathrm{Xu}$ \& Chance, 2007). Similarly, additives such as glycerol, ATP, EDTA and ADP have also been shown to significantly prolong the lifetime in footprinting experiments through quenching effects even at low concentrations: $1.0 \mathrm{~m} M$ glycerol can diminish the rate of radiolysis by a factor of five. It might therefore be expected that the lifetime of protein crystals varies significantly as a function of solvent composition. This has been demonstrated though the success of radical scavengers at room temperature in comparison to $100 \mathrm{~K}$ (Barker $e t$ al., 2009; De la Mora et al., 2011; Kmetko et al., 2011). This is also reflected in the variation in the lifetime of protein crystals at RT as a function of both solvent composition and protein type, in contrast to $100 \mathrm{~K}$ where several studies determining crystal lifetime have shown remarkable agreement (Howells et al., 2009). The increased lifetime of Fc $\gamma$ RIIIa $\left(d_{\mathrm{e}} \simeq 0.24 \mathrm{MGy}\right)$ crystals that we observe at low frame rates in comparison to $\mathrm{BEV} 2\left(d_{\mathrm{e}} \simeq 0.1 \mathrm{MGy}\right)$ and adenosine $\mathrm{A}_{2 \mathrm{~A}} \mathrm{AR}$ $\left(d_{\mathrm{e}} \simeq 0.11 \mathrm{MGy}\right)$ may in part arise from the presence of citrate, a known $\cdot \mathrm{OH}$ quencher (Gupta et al., 2007). None of the buffers characterized as efficient $\cdot \mathrm{OH}$ scavengers are present in the crystallization conditions of BEV 2 or $\mathrm{A}_{2 \mathrm{~A}} \mathrm{AR}$.

Figs. 1(b) and 2 show that for exposure times of $>100 \mathrm{~ms}$ ( $<10 \mathrm{fps})$ the Fc $\gamma$ RIIIa and BEV 2 lifetimes are independent of dose rate, suggesting that radical formation and diffusion are complete within these timescales (the limited availability of $A_{2 A} A R$ crystals meant that only a single regime with an exposure time greater than $100 \mathrm{~ms}$ could be probed). Lifetime then increases with dose rate. In all of the systems studied the fact that the mean lifetime continually increases as a function of dose rate over a number of images of $100 \mathrm{~ms}$ or less is consistent with radical chemistry on timescales of this order.

There are three possible origins of a dose-rate effect on these timescales: self-recombination of radical species, the rate of diffusion of radicals through the solvent and quenching (radical absorption) within the bulk solvent.

Firstly, there may be significant radical recombination at high flux densities. In the experiments described here the primary yield of hydroxyl and other radicals is high: for every $100 \mathrm{eV}$ absorbed $2.87 . \mathrm{OH}$ radicals are produced. Our maximum dose rate of $\sim 1 \mathrm{MGy} \mathrm{s}^{-1}$ results in the generation of approximately $1 \times 10^{5} \cdot \mathrm{OH}$ radicals, and a similar number of holes and aqueous electrons, per unit cell per second, or $\sim 4 \times 10^{3}$ per image at $25 \mathrm{~Hz}$. These radicals are not generated uniformly in the bulk solvent, but in regions a few nanometres in size called spurs (Hill \& Smith, 1994). Recombination occurs when spurs overlap. It is therefore possible that on increasing the dose rate increased radical recombination occurs, reducing the number of radicals available to damage the protein lattice.

Secondly, radicals formed in the bulk solvent will take a finite time to diffuse in sufficient numbers to cause damage observable through diffraction. In this case, a lag phase reflects the time taken for the diffusion of $\cdot \mathrm{OH}$ through the bulk solvent. In line with the rate of diffusion being a limiting factor, the increase in atomic $B$ factor has been observed to be greater close to solvent channels in thermolysin crystals at $160 \mathrm{~K}$, with residue depth conferring a degree of protection from radiation damage (Juers \& Weik, 2011).

Thirdly, radicals are quenched within the solvent. Dose-rate effects on timescales of less than $100 \mathrm{~ms}$ are consistent with the lag of $30 \mathrm{~ms}$, followed by rapid decay, observed in synchrotron footprinting experiments (Gupta et al., 2007). This lag has been attributed to quenching of $\cdot \mathrm{OH}$ by buffer molecules. In addition, as detailed earlier, solvent composition has also been found to have a significant effect on the rate of cleavage, highlighting the effect of quenching.

These observations, and those illustrating residue sensitivity changing as a function of solvent accessibility, suggest solvent quenching and diffusion delay are significant effects and a probable cause of a dose-rate effect in RT synchrotron millisecond X-ray crystallography. However, it will only be possible to fully establish which processes dominate when advances in detector technology make much shorter timescales accessible. If diffusion and quenching dominate, the observation of an $\sim 50 \%$ reduction in Fc $\gamma$ RIIIa lifetime when a pause is introduced during the experiment (Fig. 1a) can be simply explained by the increased movement of main-chain fragments disrupting the crystal lattice and the diffusion of remaining radicals through the lattice. This slow lattice disruption is likely to be the origin of the radiation-damage dark progression previously observed on the second-minute timescale above $180 \mathrm{~K}$ (Warkentin et al., 2011, 2012). On shorter timescales, and above $\sim 160 \mathrm{~K}$ when $\cdot \mathrm{OH}$ becomes mobile, radical diffusion is the origin of a dose-rate effect. The increased crystal lifetime observed at $4 \mathrm{~s}$ during continuous data collection again points to diffusion and quenching as important effects. During continuous data collection the dose is deposited in the crystal over a longer time period; radical recombination should therefore be greatly reduced, with a concomitant decrease in crystal lifetime. The increase in lifetime observed suggests that in this regime recombination plays a minor role compared with quenching and diffusion. At higher dose rates recombination of radical species will play an increasingly important role, but with the data currently available it is not possible to establish whether this or quenching and diffusion within the solvent is the origin of the dose-rate effect observed in these experiments.

If this model of diffusion, quenching and recombination of radicals resulting in increased lifetimes during continuous data collection and at high dose-rates is correct, it not only explains our results but also suggests that massive gains in crystal lifetime might be achieved with greater flux densities. This would allow many more data to be collected during an initial 'lag phase' lasting a few tens of milliseconds while significant 
radical recombination occurs and before large numbers of radicals can diffuse through the solvent resulting in rapid crystal decay. In the limit, it might be possible to approach the two-orders-of-magnitude increase in crystal lifetime achieved by cryocooling to $100 \mathrm{~K}$. Given the attractions of in situ data collection even in the face of the present highly fragmented data-collection regimes (RT data sets for large viruses have typically required many hundreds of crystals; Grimes et al., 1998; Abrescia et al., 2004, 2008), this would be likely to transform the practice of RT macromolecular crystallography.

In the short term, development of data-collection strategies to take advantage of the possibilities of fast-readout PADs combined with a microcalibre 'machine-gun' approach to depositing radiation at very high dose rates into small regions of larger crystals might prove effective. The dependence of the RT crystal lifetime on radical quenching and diffusion means that it is also worthwhile exploring the optimization of radical quenching buffers, possibly adding to the current highly successful crystallization screens to build in this added value for RT crystallography. In the medium term, we note that the next generation of cryo- and superconducting undulators promises a significant increase (up to an order of magnitude at $12 \mathrm{keV}$ ) in photon flux from the current generation of light sources, whilst the trend towards faster read-out detectors promises to deliver matching increases in performance. In the light of our experiences, described here and in Axford (2012), we believe that it will also be worthwhile devoting greater effort to optimizing data analysis from the fragmentary data which are likely to be acquired by the increased application of the so-called 'American Method' of 'shoot first and ask questions later' (Rossmann \& Erickson, 1983). For the cases of data collection for structure determination of BEV 2 and Fc $\gamma$ RIIIa (Axford et al., 2012), data collection and analysis was based on practices established through experience and was effective but very labour-intensive. In the case of the structure solution of two polymorphs of Fc $\gamma$ RIIIa (F158V) a total of $260^{\circ}$ of data were collected from each crystal type, with the number of crystals required varying between $44(158 \mathrm{~F})$ and 72 (158V) (Axford et al., 2012). Diffraction data were collected in a suboptimal fashion (given the results in this present work) with an exposure time of $0.1 \mathrm{~s}$. Taking advantage of the dose-rate effect observed in Fc $\gamma$ RIIIa (Fig. 2) would have permitted the same amount of data to be collected from $30(158 \mathrm{~F})$ and $50(158 \mathrm{~V})$ crystals: a significant reduction. Increases in incident flux and detector read-out speed promise further gains. In the meantime such protein and virus data collection is feasible, but major challenges remain in increasing the level of automation and improving the robustness of data-integration programs so that they can cope routinely with thin wedges of very weak fine-sliced data.

\section{Conclusions}

The data presented here provide clear evidence for a dose-rate effect in RT crystallography when using the full flux of an undulator beamline. The effect becomes significant at exposure times less than $60 \mathrm{~ms}$ (frame rates of $>16 \mathrm{~Hz}$ ), with life- times increasing as a function of dose rate. Also apparent is a reduced crystal lifetime when there is a pause between frames within a data set when, for example, the X-ray detector is read out. Both of these observations can be explained by considering a three-part model for radiation damage at room temperature. On slow timescales $(>1 \mathrm{~s})$ radical diffusion and quenching within the solvent occur, while on fast timescales $(<60 \mathrm{~ms})$ radical diffusion, quenching and recombination are relevant. Differences in free-radical formation and propagation at RT, the latter of which is quite different between RT and $100 \mathrm{~K}$, account for the absence of a significant dose-rate effect in cryocrystallography. These observations suggest that more intense beams and faster detectors might render RT data collection a generally attractive strategy for the collection of macromolecular crystallography data.

\section{APPENDIX $A$ \\ Count-rate correction}

The Pilatus $6 \mathrm{M}$ is a paralyzable detector and as such a deadtime correction must be made at high count rates. The correction is of the form $C=C_{0} \exp \left(-C_{0} \tau\right)$, where $C_{0}$ is the measured count rate, $C$ is the corrected count rate and $\tau$ is the dead time. For the experiments carried out here the dead time was $199 \mathrm{~ns}$. This correction is applied by the detector software. The combination of the high fluxes and short exposure times used in this study mean that it is important to confirm that the correction made is valid, as if it were not the effect would be to underestimate intense reflections, distorting the intensity decay curves. Supplementary Fig. S1 shows the count-rate correction curve for the I24 Pilatus 6M. The $y$ axis is marked at the maximum count rate recommended by the manufacturer and at the maximum count rate observed for each of the systems included in this work. It is clear from the plot that the experiments carried out here are well within the valid range of the dead-time correction model.

\section{APPENDIX $B$ \\ UV-Vis of irradiated water}

UV-Vis absorption data were also collected from thin films of pure water at I24. Several factors combine to make the collection of low-wavelength $(<250 \mathrm{~nm})$ data difficult: the presence of microcrystalline ice makes spectra noisy, the intensity of the deuterium halogen lamp is low in this wavelength region and optical losses become large. Nonetheless, a clear absorbance shoulder was observed to form at the lowwavelength limit (Supplementary Fig. S2) upon exposure to $\mathrm{X}$-rays, indicating the formation of hydroxyl radicals (Janik et al., 2007). We believe this to be a shoulder rather than a distinct band centred at $280 \mathrm{~nm}$ owing to the instrument and sample limitations outlined above. Such a shoulder is in agreement with previous work on the radiolysis of pure water. Despite these limitations and the relatively weak absorbance of the hydroxyl radical, it is clear that an X-ray-induced change in absorbance is readily observed using the Diamond 
microspectrophotometer. It should be noted that the presence of cryoprotectants in the solutions studied in Fig. 4 greatly reduces sample limitations (with respect to UV-Vis absorption spectroscopy), with the result that the low-wavelength limit is lowered.

We would like to thank members of the Diamond MX and data-acquisition groups, in particular Jun Aishima, for assistance with the experiments, and Julien Marchal for useful discussions. We would also like to thank Ian Carmichael and Elspeth Garman for useful discussions on free radicals. We would also like to thank Professor D. Rowlands (University of Leeds) for providing samples of BEV 2. The Division of Structural Biology at the University of Oxford is supported by the MRC.

\section{References}

Abrescia, N. G., Cockburn, J. J., Grimes, J. M., Sutton, G. C., Diprose, J. M., Butcher, S. J., Fuller, S. D., San Martín, C., Burnett, R. M., Stuart, D. I., Bamford, D. H. \& Bamford, J. K. (2004). Nature (London), 432, 68-74.

Abrescia, N. G., Grimes, J. M., Kivelä, H. M., Assenberg, R., Sutton, G. C., Butcher, S. J., Bamford, J. K., Bamford, D. H. \& Stuart, D. I. (2008). Mol. Cell, 31, 749-761.

Axford, D. et al. (2012). Acta Cryst. D68, 592-600.

Barker, A. I., Southworth-Davies, R. J., Paithankar, K. S., Carmichael, I. \& Garman, E. F. (2009). J. Synchrotron Rad. 16, 205-216.

Bingel-Erlenmeyer, R., Olieric, V., Grimshaw, J. P. A., Gabadinho, J., Wang, X., Ebner, S. G., Isenegger, A., Schneider, R., Schneider, J., Glettig, W., Pradervand, C., Panepucci, E. H., Tomizaki, T., Wang, M. \& Schulze-Briese, C. (2011). Cryst. Growth Des. 11, 916-923.

Blake, C. \& Phillips, D. (1962). Proceedings of the Symposium on the Biological Effects of Ionising Radiation at the Molecular Level, pp. 183-191. Vienna: International Atomic Energy Agency.

Blundell, S. \& Johnson, L. (1976). Protein Crystallography. New York: Academic Press.

Brivati, J. A., Symons, M. C. R., Tinling, D. J. A. \& Williams, D. O. (1969). J. Chem. Soc. A, pp. 719-720.

Buxton, G. V. (1987). Radiation Chemistry: Principles and Applications, edited by Farhataziz \& M. A. J. Rodgers, pp. 321-348. New York: VCH.

Chapman, H. N. et al. (2011). Nature (London), 470, 73-77.

Cherezov, V., Riedl, K. M. \& Caffrey, M. (2002). J. Synchrotron Rad. 9, 333-341.

De la Mora, E., Carmichael, I. \& Garman, E. F. (2011). J. Synchrotron Rad. 18, 346-357.

Dorfman, L. \& Adams, G. (1973). Reactivity of the Hydroxyl Radical in Aqueous Solutions. Washington: US National Bureau of Standards.

Eikenberry, E. F., Brönnimann, C., Hülsen, G., Toyokawa, H., Horisberger, R., Schmitt, B., Schulze-Briese, C. \& Tomizaki, T. (2003). Nucl. Instrum. Methods A, 501, 260-266.

Fraser, J. S., van den Bedem, H., Samelson, A. J., Lang, P. T., Holton, J. M., Echols, N. \& Alber, T. (2011). Proc. Natl Acad. Sci. USA, 108, 16247-16252.

Garman, E. F. (2010). Acta Cryst. D66, 339-351.

Garman, E. \& Owen, R. L. (2007). Methods Mol. Biol. 364, 1-18.

Grimes, J. M., Burroughs, J. N., Gouet, P., Diprose, J. M., Malby, R., Ziéntara, S., Mertens, P. P. \& Stuart, D. I. (1998). Nature (London), 395, 470-478.

Gupta, S., Sullivan, M., Toomey, J., Kiselar, J. \& Chance, M. R. (2007). J. Synchrotron Rad. 14, 233-243.
Hill, M. A. \& Smith, F. A. (1994). Radiat. Phys. Chem. 43, 265280.

Howells, M. R., Beetz, T., Chapman, H. N., Cui, C., Holton, J. M., Jacobsen, C. J., Kirz, J., Lima, E., Marchesini, S., Miao, H., Sayre, D., Shapiro, D. A., Spence, J. C. H. \& Starodub, D. (2009). J. Electron Spectrosc. Relat. Phenom. 170, 4-12.

Hug, G. (1981). J. Phys. Chem. A, 111, 1835-1843.

Ivanova, E., Suvorov, O. \& Yudin, I. (2009). High Energ. Chem. 43, 263-268.

Jacquamet, L., Ohana, J., Joly, J., Borel, F., Pirocchi, M., Charrault, P., Bertoni, A., Israel-Gouy, P., Carpentier, P., Kozielski, F., Blot, D. \& Ferrer, J.-L. (2004). Structure, 12, 1219-1225.

Janik, I., Bartels, D. M. \& Jonah, C. D. (2007). J. Phys. Chem. A, 111, 1835-1843.

Jones, G. D., Lea, J. S., Symons, M. C. \& Taiwo, F. A. (1987). Nature (London), 330, 772-773.

Juers, D. H. \& Weik, M. (2011). J. Synchrotron Rad. 18, 329-337.

Klassen, N. (1987). Radiation Chemistry: Principles and Applications, edited by Farhataziz \& M. A. J. Rodgers, pp. 29-64. New York: $\mathrm{VCH}$.

Kmetko, J., Warkentin, M., Englich, U. \& Thorne, R. E. (2011). Acta Cryst. D67, 881-893.

Lebon, G., Warne, T., Edwards, P. C., Bennett, K., Langmead, C. J., Leslie, A. G. \& Tate, C. G. (2011). Nature (London), 474, 521525.

Mao, W. L., Mao, H., Goncharov, A. F., Struzhkin, V. V., Guo, Q., Hu, J., Shu, J., Hemley, R. J., Somayazulu, M. \& Zhao, Y. (2002). Science, 297, 2247-2249.

McGeehan, J., Ravelli, R. B. G., Murray, J. W., Owen, R. L., Cipriani, F., McSweeney, S., Weik, M. \& Garman, E. F. (2009). J. Synchrotron Rad. 16, 163-172.

Meents, A., Gutmann, S., Wagner, A. \& Schulze-Briese, C. (2010). Proc. Natl Acad. Sci. USA, 107, 1094-1099.

Neutze, R., Wouts, R., van der Spoel, D., Weckert, E. \& Hajdu, J. (2000). Nature (London), 406, 752-757.

Owen, R. L., Yorke, B. A., Gowdy, J. A. \& Pearson, A. R. (2011). J. Synchrotron Rad. 18, 367-373.

Paithankar, K. S. \& Garman, E. F. (2010). Acta Cryst. D66, 381-388.

Paithankar, K. S., Owen, R. L. \& Garman, E. F. (2009). J. Synchrotron Rad. 16, 152-162.

Rajendran, C., Dworkowski, F. S. N., Wang, M. \& Schulze-Briese, C. (2011). J. Synchrotron Rad. 18, 318-328.

Rossmann, M. G. \& Erickson, J. W. (1983). J. Appl. Cryst. 16, 629-636.

Sauter, N. K. \& Poon, B. K. (2010). J. Appl. Cryst. 43, 611-616.

Sclavi, B., Woodson, S., Sullivan, M., Chance, M. R. \& Brenowitz, M. (1997). J. Mol. Biol. 266, 144-159.

Snell, E. H., Bellamy, H. D., Rosenbaum, G. \& van der Woerd, M. J. (2007). J. Synchrotron Rad. 14, 109-115.

Southworth-Davies, R. J. \& Garman, E. F. (2007). J. Synchrotron Rad. 14, 73-83.

Southworth-Davies, R. J., Medina, M., Carmichel, I. \& Garman, E. (2008). Structure, 12, 1531-1541.

Swartz, H. M. \& Swartz, S. M. (1983). Methods Biochem. Anal. 29, 207-323.

Symons, M. (1995). Radiat. Phys. Chem. 45, 837-845.

Symons, M. (1999). Prog. React. Kinet. Mech. 24, 139-164.

Takamoto, K. \& Chance, M. R. (2006). Annu. Rev. Biophys. Biomol. Struct. 35, 251-276.

Walter, T. S. et al. (2005). Acta Cryst. D61, 651-657.

Warkentin, M., Badeau, R., Hopkins, J. B., Mulichak, A. M., Keefe, L. J. \& Thorne, R. E. (2012). Acta Cryst. D68, 124-133.

Warkentin, M., Badeau, R., Hopkins, J. \& Thorne, R. E. (2011). Acta Cryst. D67, 792-803.

Xu, G. \& Chance, M. R. (2007). Chem. Rev. 107, 3514-3543.

Zakurdaeva, O. A., Nesterov, S. V. \& Feldman, V. I. (2005). High Energ. Chem. 39, 201-206. 